\title{
MICROFABRICATION AND TEST OF AN INTEGRATED COLLOID THRUSTER
}

\author{
R. Krpoun, M. Räber and H.R. Shea \\ Microsystems for Space Technologies Laboratory (LMTS) \\ Ecole Polytechnique Fédérale de Lausanne (EPFL), Switzerland
}

\begin{abstract}
This paper reports on the design, fabrication and test of an integrated colloid micropropulsion system for spacecraft attitude control using the ionic liquid EMI-BF as fuel. The principle of operation of the thruster is identical to electrospray ionization. The objective of the project was to demonstrate the feasibility and operability of arrays of microfabricated capillary emitters with individual extractor electrodes. This design approach results in an identical electric field distribution from one capillary to the other, avoids crosstalk and therefore allows for a more finely modulated thrust control. Spraying tests with different thruster configurations were conducted under vacuum conditions. Tests were performed with different thruster configurations and starting voltages around $700 \mathrm{~V}$ were observed.
\end{abstract}

\section{INTRODUCTION}

Colloid thrusters operate by electrically charging a conductive liquid at the tip of a capillary to high voltage. Above a critical voltage the liquid deforms into a cone and an aerosol of charged droplets or ions is accelerated towards an extractor electrode, as shown in figure 1. For spacecraft attitude control thrust in the micro- to millinewton range is required. As the thrust per emitter is in the $0.1 \mu \mathrm{N}$ range arrays of emitters are necessary. The objective of this project is to demonstrate the feasibility and operability of arrays of microfabricated capillary emitters with integrated extractor electrodes.

Prior work on out-of-plane electrospray emitters without extraction electrodes has been carried out by Schultz et al. [1], Griss at al. [2] and Wang et al. [3]. Pioneering work on integrated capillary devices has been done by Paine et al. [4], [5], unfortunately the low aspect ratio of his capillaries led to fuel leakage and his devices were not functional. Xiong et al. [6] have demonstrated the feasibility of an integrated device with starting voltages of $1400 \mathrm{~V}$, twice the voltage required for our

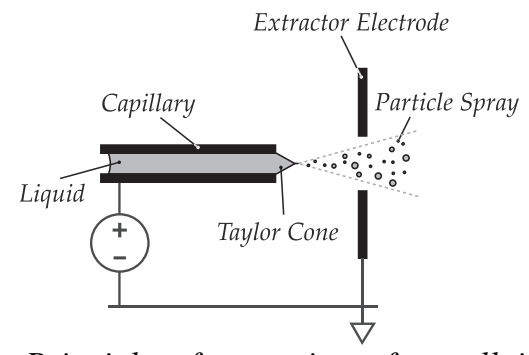

Figure 1: Principle of operation of a colloid thruster. Droplets and ions are extracted from a conductive liquid by applying a potential difference between the liquid and the extractor electrode. devices. One should also mention the work of VelásquezGarcía et al. [7] and Gassend [8] who studied integrated electrospray thrusters using externally wetted cones rather than capillaries.

For our experiments the ionic liquid EMI- $\mathrm{BF}_{4}$ is used which seems particularly suited for application in colloid thrusters as it has no measurable vapor pressure and spraying of ions, ion complexes as well as droplets has been demonstrated [9], [10].

The novelty of this work lays in the combination of high aspect ratio capillary emitters (height of $70 \mu \mathrm{m}$, inner diameter of $20 \mu \mathrm{m})$ with individual extractor electrodes having diameters from $80 \mu \mathrm{m}$ upwards and a spacing as low as $25 \mu \mathrm{m}$ from the capillary tips. An overview of the geometry is shown in figure 2. Our experiments show that with high aspect ratio capillaries so close to the extractor electrodes the critical electrical field for spraying is reached at much lower voltages than observed for other electrospray thrusters. The individual integrated electrodes, as opposed to the standard approach of one common extractor electrode for multiple capillary emitters, allow for very high uniformity in critical voltages between capillaries and hence more finely modulated thrust control. Finally the current-voltage results presented in this paper have all been obtained without applying a pressure to the liquid, pumping occurred only passively by capillarity.

\section{FABRICATION}

Microfabrication of the thruster is divided into manufacturing capillary arrays and extractor electrodes. For vacuum testing the thruster is assembled onto a printed circuit board (PCB) that serves as mechanical support. Figure 3 shows a schematic cross section of the thruster test assembly.

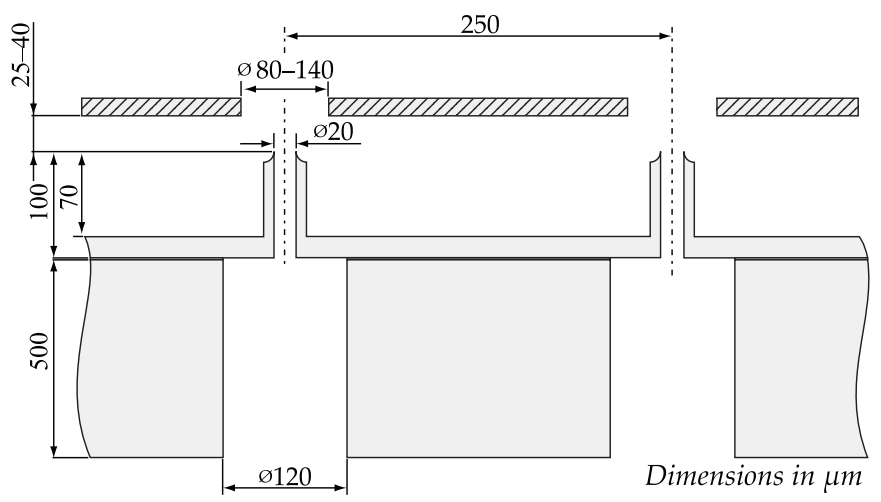

Figure 2: Geometry of the microfabricated thruster. The design includes variations in the extractor electrode diameter, and the distance between capillaries and the extractor. 


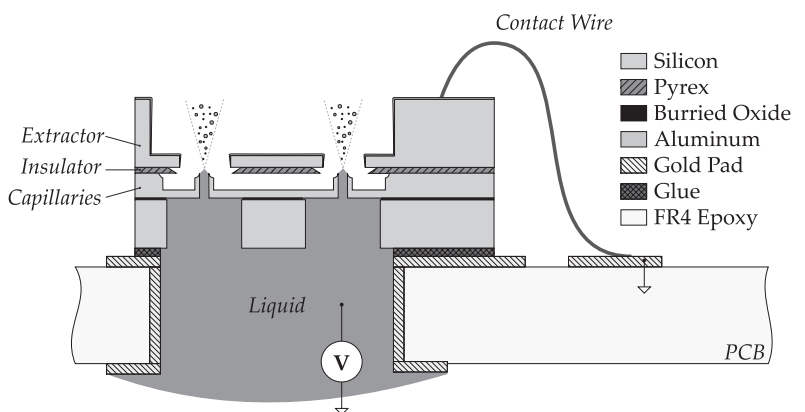

Figure 3: Schematic cross-section of the integrated thruster. The microfabricated thruster elements are mounted onto a PCB that serves as support during testing.

$$
\begin{aligned}
& \square \text { Photoresist } \square \text { Thermal Oxide } \\
& \text { CVD Oxide } \square \text { Silicon } \\
& \square \text { Nitride }
\end{aligned}
$$

a)

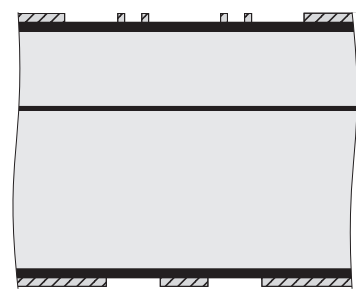

b)

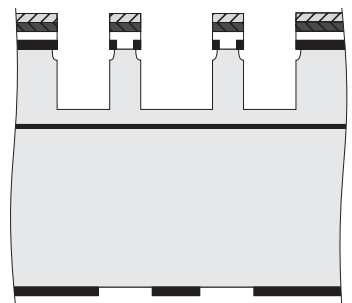

c)
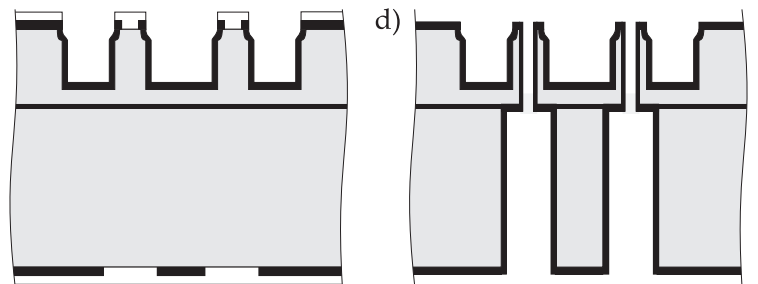

Figure 4: Fabrication process of the capillary emitters. The $70 \AA$ thick buffer oxide is not shown

\section{Capillary Emitters}

The process flow for the capillary arrays is summarized in figure 4 and is inspired by a design presented by Griss et al. [2], modified to allow the manufacture of large capillary arrays with flat tips. The fabrication process starts with steam oxidation of a silicon-on-insulator (SOI) wafer with a $100 \mu \mathrm{m}$ device layer, $1 \mu \mathrm{m}$ buried oxide and a $500 \mu \mathrm{m}$ handle layer. After photolithography using AZ1518 photoresist the exposed fractions of the $1.5 \mu \mathrm{m}$ thick oxide are removed in a buffered HF solution (a). This oxide layer defines the standoff structures which will protect the capillaries and allow bonding of the extractor electrodes during assembly. Next a thin buffer oxide is grown acting as stress relief for a subsequent 150nm LPCVD nitride layer. The latter serves as local protection of the silicon during steam oxidation. Finally an additional CVD oxide is grown that serves as a hard mask. This oxide-nitrideoxide structure is patterned by reactive ion etching using an AZ1518 photoresist as mask. The following isotropic RIE and deep reactive ion etch (DRIE) yield the characteristic outer shape of the capillaries $(b)$. After

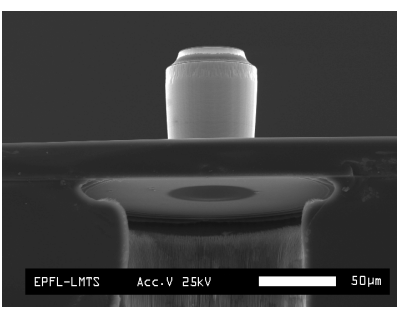

(a)

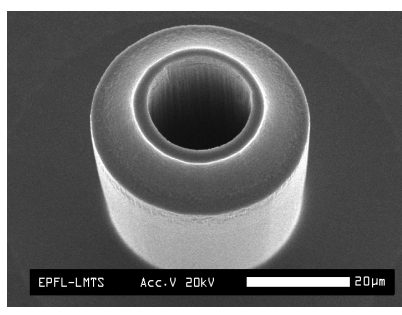

(b)
Figure 5: SEM photographs of the capillaries: (a) cross section of a capillary before HF vapor etch. Notching at the buried oxide is clearly visible. (b) tip of a capillary with $20 \mu \mathrm{m}$ inner diameter.

photoresist stripping and CVD oxide removal the wafers are oxidized $(c)$. The resulting $1.5 \mu \mathrm{m}$ thick oxide serves as mask during the second DRIE which is performed once the nitride has been removed in a phosphoric acid bath. This DRIE etches the inner hole of the capillaries. Once micromachining of the front side is achieved the back side of the wafer is etched up to the buried oxide using a combined oxidephotoresist mask (AZ4562). Finally the buried oxide is removed by means of a HF vapor etch [11] and the whole wafer oxidized to improve wetting.

\section{Extractor Electrodes}

The two major constraints driving the design of the extractor electrode are the electric isolation between capillary emitters and extractor electrodes and the large beam half angle. To avoid breakdown at the required extraction voltages $(\mathrm{kV})$, the insulator should be as thick as possible, at least tens of microns. To allow for high capillary density, and to avoid spraying onto the extraction electrodes due to the beam halfangle of typically $30^{\circ}$, the sum of extractor and insulator thicknesses should minimized. We chose a minimum emitter pitch of 250 microns, which then dictated maximum thicknesses.

The process flow in which the silicon acts as extractor electrode and etch mask [12] is shown in figure 6. The 50um deep electrodes are etched into a $500 \mu \mathrm{m}$ thick silicon wafer by DRIE. A Borofloat wafer with a thickness of $250 \mu \mathrm{m}$ is then anodically bonded to the silicon wafer $(i)$. To allow a dense packing of the emitters the Borofloat wafer is thinned down to $80 \mu \mathrm{m}$ in a HF $20 \%$ bath and the backside is machined in a second DRIE (ii). Finally a thin layer of aluminum is deposited that improves the electric contacts during final assembly (iii). (i)

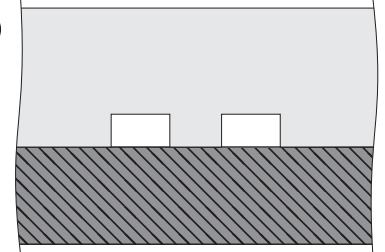

שू Aluminium

$\triangle$ Borofloat

$\square$ Silicon (ii)

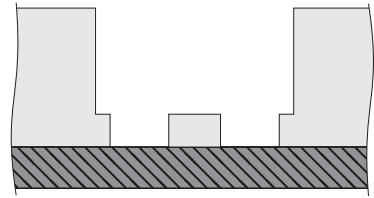

(iii)

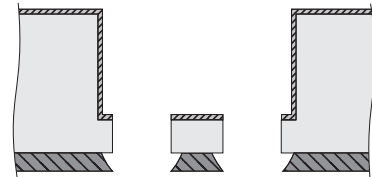

Figure 6: Process flow of the extractor electrodes. 


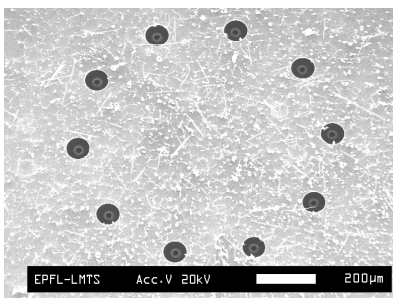

(a)

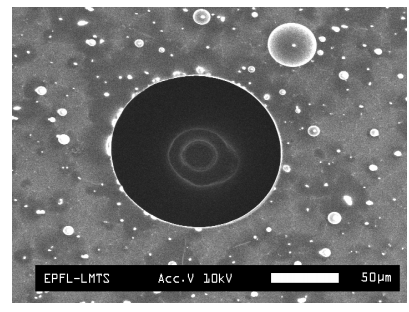

(b)
Figure 6: SEM photographs of the assembled thruster. (a) overview of an array. $(b)$ detail of a capillary after spray tests. Droplets, probably reflected by the retarding grid, are clearly visible.

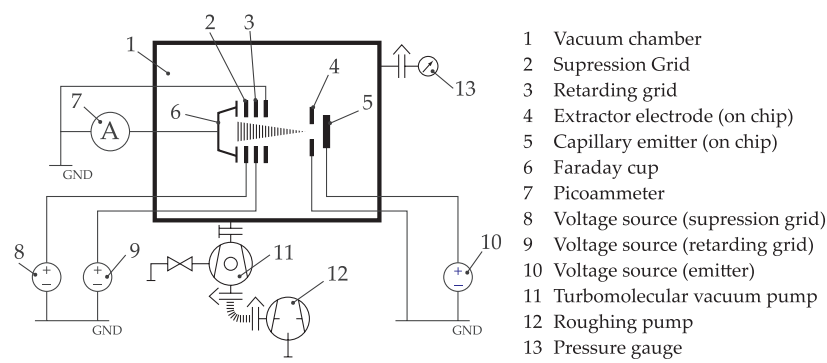

Figure 7: Schematic of the electrospray test rig.

As it is problematic to obtain a homogeneous backside etch over the whole 4" wafer, figure 6 , the same process flow is currently under investigation using SOI wafers to improve the surface quality of the extractor electrodes.

\section{Assembly}

Once microfabrication is complete the wafers are diced and the chips assembled by gluing extractor and capillaries together. Two alignment methods were investigated: The first, manually aligning the chips under a microscope by means of a multi-axis positioning stage, yields an alignment accuracy in the range of $\pm 5 \mu \mathrm{m}$. The second approach has been to include groves on the chips into which ruby balls of $120 \mu \mathrm{m}$ or $200 \mu \mathrm{m}$ diameter are placed. Although this method was only attempted on a few chips, as groves had to be etched into the Borofloat using plasma etching, the observed results are superior to manual alignment. Future developments will include passive alignment features for chip level assembly and anodic bonding for wafer scale assembly.

\section{EXPERIMENTAL SETUP}

Beam current and energy were measured to verify the functionality of the microfabricated devices. Spraying was carried out at pressures below $1 \times 10^{-5} \mathrm{mbar}$ to simulate space vacuum. Figure 7 shows a schematic of the test setup consisting of a vacuum chamber, high voltage sources, a Faraday cup for current measurement and a retarding potential grid to determine beam energy. The

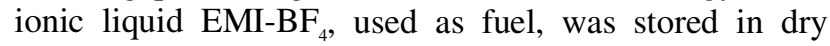
conditions to avoid contamination.

\section{RESULTS}

Measurements were carried out with various thruster configurations and are described in more detail in [13]. Figure 8 shows a current-voltage curve of a single capillary, with $40 \mu \mathrm{m}$ emitter-extractor distance, a capillary inner diameter of $20 \mu \mathrm{m}$ and $140 \mu \mathrm{m}$ diameter extractor. The measurements were done by first increasing and then decreasing the voltage until spray extinction, $V_{o x}$. The measured behavior and intensity of spray current, in particular the characteristic local minimum, correlates with current-voltage measurements reported in literature for capillary pumped [10] and forced liquid flow [9].

Figure 9 shows the current-voltage curve for two emitters with different extractor electrodes having $115 \mu \mathrm{m}$ and $140 \mu \mathrm{m}$ diameter. As the voltage increases the first emitter starts spraying at $700 \mathrm{~V}$, as the voltage is further increased a jump in current is observed which indicates the emission starting from the second capillary. As future spacecraft require thrust ranging from micro- to millinewton this difference in starting voltages could be employed to generate coarse thrust modulation.

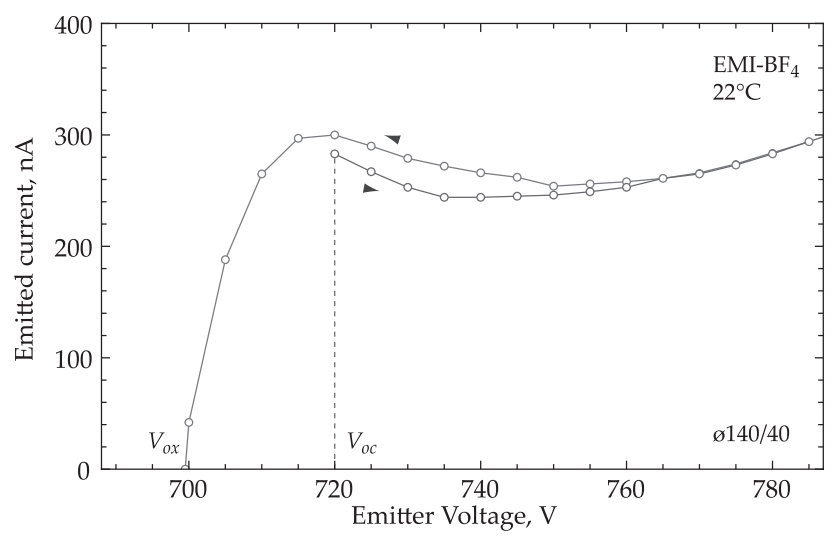

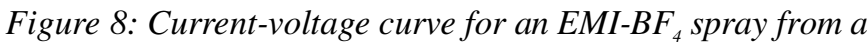
capillary with $20 \mu \mathrm{m}$ i.d. and a $140 \mu \mathrm{m}$ diameter electrode spaced $40 \mu \mathrm{m}$ from the emitter.

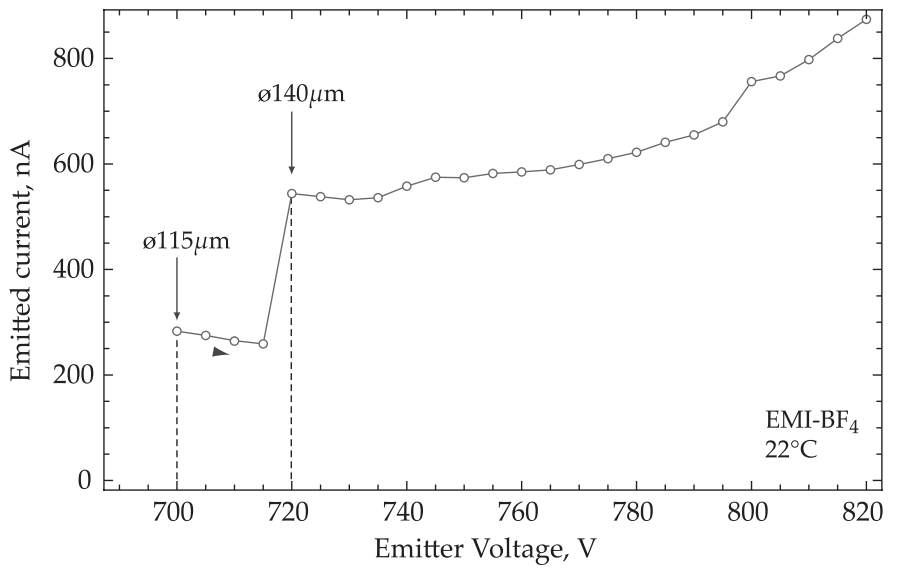

Figure 9: Current-voltage curve for $\mathrm{EMI}-\mathrm{BF}_{4}$ of two capillaries with $20 \mu \mathrm{m}$ i.d. with the extraction electrode spaced $40 \mu m$ from the emitters. The jump in current is due to the different starting voltages for an extractor electrode with $115 \mu \mathrm{m}$ and one with $140 \mu \mathrm{m}$ diameter. 


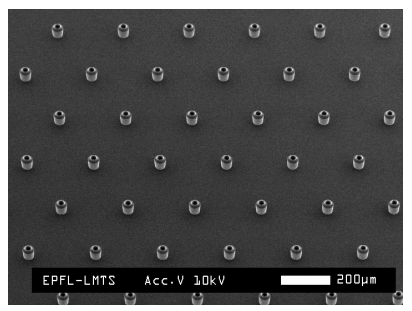

(a)

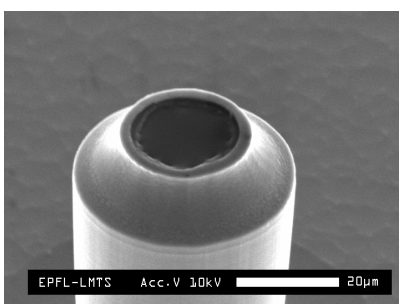

(b)
Figure 10: SEM photographs of the capillaries. (a) array of emitters. (b) capillary filled with $E M I-B F_{4}$ before spray tests.

We have observed that spray current could only be obtained for extractor diameters above $115 \mu \mathrm{m}$ as extractors with smaller diameter holes were quickly jammed by the large droplets in the spray. No test results are reported for large arrays as they were designed with $80 \mu \mathrm{m}$ extractor hole diameter. Large droplets have been observed on top of the extractor electrode, as is shown in figure 6. Their exact provenance is still unclear, but it is likely that they were reflected back to the extraction electrode during retarding potential measurements.

\section{CONCLUSION}

A colloid microthruster prototype has been designed, fabricated and tested. The developed microfabrication process yields large arrays of capillary emitters and extractor electrodes with well defined geometries. The operation of the devices for several geometries has been experimentally demonstrated. Beam currents in the order of 300nA have been measured with starting voltages around $700 \mathrm{~V}$ and a method for coarse thrust modulation by varying extractor electrode diameters.

\section{ACKNOWLEDGEMENTS}

The authors would like to thank Prof. J.P.W. Stark and K.L. Smith from Queen Mary, University of London for their valuable inputs during the development of the presented prototype. We further like to acknowledge the partial support from the European Space Agency and thank the staff of the EPFL-CMI and the University of Neuchâtel COMLAB for their help during device fabrication.

\section{REFERENCES}

[1] G.A. Schultz, T.N. Corso, S.J. Prosser and S. Zhang, "A fully integrated monolithic microchip electrospray device for mass spectrometry," Anal. Chem., vol. 72, pp. 4058-4063, 2000.
[2] P. Griss, J. Melin, J. Sjödahl, J. Roeraade and G. Stemme, "Development of micromachined hollow tips for protein analysis based on nanoelectrospray ionization mass spectrometry," J. Micromech. Microeng., vol. 12, pp. 682-687, 2002.

[3] L. Wang, R. Stevens, A. Malik, P. Rockett, M. Paine, P. Adkin, S. Martyn, K. Smith, J. Stark and P. Dobson, "High-aspect-ratio silica nozzle fabrication for nanoemitter electrospray applications," Microelectron. Eng., vol. 84, pp. 1190-1193, 2007.

[4] M.D. Paine, S. Gabriel, C.G.J. Schabmueller and A.G.R. Evans, "Realisation of very high voltage electrode-nozzle systems for MEMS," Sens. Actuators, A, vol. 114, pp. 112-117, 2004.

[5] M.D. Paine, "A micro-fabricated colloid micro-thruster," $\mathrm{PhD}$ thesis, University of Southampton, 2002.

[6] J. Xiong, Z. Zhou, D. Sun and X. Ye, "Development of a MEMS based colloid thruster with sandwich structure," Sens. Actuators, A, vol. 117, pp. 168-172, 2005.

[7] L.F. Velásquez-García, A.I. Akinwande and M. Martínez-Sánchez, "A planar array of micro-fabricated electrospray emitters for thruster applications," $J$. Microlectromech. Syst., vol. 15, pp. 1272-1280, 2006.

[8] B.L.P. Gassend, "A fully microfabricated twodimensional electrospray array with applications to space propulsion," $\mathrm{PhD}$ thesis, Massachusetts Institute of Technology, 2007.

[9] I. Romero-Sanz, R. Bocanegra and J. Fernandez de la Mora, "Source of heavy molecular ions based on Taylor cones of ionic liquids operating in the pure ion evaporation regime," J. Appl. Phys., vol. 94, pp. 35993605, 2003.

[10] S. Jhuree, M.S. Alexander and J.P.W. Stark, "Electrostatic influence on charge to mass spectrum in mixed ion/droplet mode: implications for colloid thruster design," in Proc. International Electric Propulsion Conference 2007, Florence, paper no. 120, 2007.

[11] T. Overstolz, P.A. Clerc, W. Noell, M. Zickar and N.F. de Rooij, "A clean wafer-scale chip-release process without dicing based on vapor phase etching," in Proc. $17^{\text {th }}$ IEEE International Conference on Micro Electro Mechanical Systems 2004, pp. 717-720, 2004.

[12] T. Corman, P. Enoksson and G. Stemme, "Deep wet etching of borosilicate glass using an anodically bonded silicon substrate as mask," J. Micromech. Microeng., vol. 8, 84-87, 1998.

[13] R. Krpoun, M. Räber, H.R. Shea, "Design and fabrication of an integrated MEMS-based colloid micropropulsion system," in Proc. International Electric Propulsion Conference 2007, Florence, paper no. 099, 2007. 